\title{
Cloning and Expression of the Serine Carboxypeptidase Gene in Zea mays and Its Antifungal Activity against Rhizoctonia solani
}

\author{
Li Liu ${ }^{1}$, Xiujing $\mathrm{He}^{1}$, Zhiming Zhang ${ }^{1}$, Maojun Zhao ${ }^{2}$, Jing Wang ${ }^{1}$, Haijian Lin ${ }^{1}$, Ya'ou Shen ${ }^{1}$ and Guangtang Pan $^{1}$ \\ 1. Maize Research Institute of Sichuan Agricultural University//Key Laboratory of Biology and Genetic Improvement of Maize in \\ Southwest Region of Chinese Ministry of Agriculture, Chengdu 611130, Sichuan, China \\ 2. College of Life and Basic Sciences, Sichuan Agricultural University, Ya'an 625014, Sichuan, China
}

Received: November 19, 2012 / Accepted: January 06, 2013 / Published: February 28, 2013.

\begin{abstract}
The authors cloned and identified a new maize serine carboxypeptidase gene named ZmSCP from R15 inbred lines seedlings which were induced by Rhizoctonia solani AG1-IA. ZmSCP encodes a 332 amino acid protein with a predicted molecular mass of $36.5 \mathrm{kDa}$ and $\mathrm{pI}$ of 4.75. Phylogenetic analysis revealed that ZmSCP showed closer kinship with Oryza sativa and sorghum, which belong to the same evolutionary branch. Amino acid sequence analysis revealed that there are four types of amino acids in ZmSCP, the percentages of them are $43.1 \%, 26.9 \%, 13.9 \%$ and $13.1 \%$. The authors subsequently purified the recombinant protein which expressed in Escherichia coli BL21 and analyzed its antimicrobial activities in vitro. Results showed that the recombinant protein inhibited hyphal growth of Rhizoctonia solani. The study suggests that the expression of ZmSCP is closely related to maize sheath blight resistance caused by Rhizoctonia solani. Further, the antifungal activity showed that ZmSCP may play at role in the disease resistance response.
\end{abstract}

Key words: Maize, serine carboxypeptidases, banded leaf and sheath blight, Rhizoctonia solani.

\section{Introduction}

SCPs (Serine carboxypeptidases) are proteins belonging to the hydrolase family $[1,2]$, mainly distributed in lysosomes of animals and vacuoles of plants, and share characteristic structural features, e.g., a signal sequence for intracellular trafficking and/or secretion, multiple N-linked glycosylation sites, and four evolutionarily conserved regions, which consist of a substrate-binding domain and three catalytic sites [3]. At present, SCPs have been isolated from several plants and crop such as Oryza sativa, Arabidopsis thaliana, Triticum aestivum, Hordeum vulgare, etc. [4-8]. Arabidopsis has at least 51 members [1], while

Corresponding author: Guang-tang Pan, Ph.D., professor, research field: crop genetics and breeding. E-mail: pangt1956@yahoo.com.cn.

Li Liu and Xiu-jing He contributed equally to this work.
71 SCPs exist in rice genome [2], indicating functional redundancy for members of the SCP family. SCPs are widely distributed in higher organisms, whereas, some of the members' functions were confirmed, such as hydrolysis of storage protein in seed [9], intracellular components autolysis in programmed cell death [10], seed development [11], trauma reactions [12], resistance to adversity [13]. However, to date, there is little research on SCP in maize.

BLSB (Banded leaf and sheath blight), caused by Rhizoctonia solani AG1-IA, is an important disease in maize worldwide. Few genes related to resistance of this pathogen have been isolated in maize, due to its large genome size, complex genome structure, and quantitative trait nature. These characteristics have hampered the ability to conduct detailed studies of 
resistance mechanisms and exploitation of resistance resources in maize. Studies on molecular mechanism of disease resistance mainly concentrated on screening of resistant QTL and cloning of resistant gene [14-16]. In previous studies, the authors have acquired large amount of genes from high tolerance maize inbred line R15 under the induction of Rhizoctionia solani using SSH (suppression subtractive hybridization method), and 35 ESTs (expression sequences tags) were identified including serine carboxypeptidases and pathogenesis-related protein [16]. In the present study, in order to investigate the gene function of ZmSCP, we isolated the ZmSCP from AG1-IA-induced high-resistance inbred maize line R15. Subsequently the authors obtained the prokaryotic expression vector pET32a $(+)-Z m S C P$ and purified the recombinant protein which was used for analysis of its antimicrobial activities in vitro. The results provide valuable information for comprehensive understanding of $\mathrm{ZmSCP}$ function.

\section{Materials and Methods}

\subsection{Plant Materials}

High-resistance maize inbred line R15 was used in the current study. Seeds were treated with $7 \%$ hypochlorite solution for $30 \mathrm{~min}$ followed by three washing with sterilized $\mathrm{H}_{2} \mathrm{O}$ before being sowed in pots with autoclaved soil. Plants were allowed to grow for 25-35 days and were then inoculated with Rhizoctonia solani (kindly provided by the Rice Institute of the Sichuan Agricultural University, Sichuan, China) at $28^{\circ} \mathrm{C}$. Rhizoctonia solani AG1-IA was cultured on potato dextrose agar and incubated at $28^{\circ} \mathrm{C}$ for 3-5 days before use.

\subsection{RNA Extraction and Cloning of ZmSCP}

Total RNA was extracted using Trizol ${ }^{\circledR}$ reagent (Invitrogen, USA) according to the manufacturer's protocol. Five hundred nanograms of DNase-treated total RNA were reverse-transcribed (TaKaRa, Dalian, China). Primers were designed according to the
ZmSCP sequence (GenBank Accession Number: JF682634): forward primer 5' $\underline{\text { GAATTC }}$ ATGCGCGGGTTCGACGGGCC3' (EcoR I site is underlined) and reverse primer 5' GCGGCCGC TTCCCCCAAGCGTAGGATAGA3' (Not I site is underlined). Amplied products were gel-separated on $1.2 \%$ agarose and extracted (Omega, China), then cloned into the pMD18-T vector (TaKaRa, Dalian, China) for sequencing. Three positive clones were sequenced. The ExPASy translate tool (http://au.expasy.org/tools/dna.html) was used to deduce the amino acid sequence from the cDNA. The amino acid compositions of the ZmSCP were carried out using DNA tool 6.0. Cluster analysis was conducted using DNAstar 7.0 to reveal evolutionary relationships of the proteins from different plants.

\subsection{Construction of Expression Plasmid and Optimization of Expression Conditions}

The pMD18-ZmSCP vector and $\mathrm{pET} 32 \mathrm{a}(+)$ were digested with EcoR I and Not I, respectively. The expected fragments were released from pMD18-ZmSCP and subcloned into pET32a $(+)$ vector using $\mathrm{T}_{4}$ DNA ligase (TaKaRa, Dalian, China). The recovered plasmid was transformed into E. coli DH5a and positive clones were selected, which were named pET-32a(+)-ZmSCP. The resulting plasmid was used to transform E. coli BL21 and was verified by sequencing. Bacteria were grown with two temperature levels at $37{ }^{\circ} \mathrm{C}$ and $28^{\circ} \mathrm{C}$ respectively to an appropriate density $\left(O D_{600}=0.6\right)$, and induced with $0,0.2,0.4,0.6,0.8$, or $1.0 \mathrm{mM}$ IPTG for $0,2,4,6$, 8 , and $10 \mathrm{~h}$ to identify optimum expression conditions.

\subsection{SDS-PAGE and Western Blot}

The expressed protein was analyzed by SDS-PAGE (sodium dodecylsulfate-polyacrylamide gel electrophoresis) and western blotting. SDS-PAGE and western blotting were carried out using standard conditions [17]. After SDS-PAGE, proteins were transferred to PVDF membranes incubated in methyl 
alcohol for $15 \mathrm{~min}$ at room temperature using the Bio-Rad trans-blot apparatus (Bio-Rad, USA) for $1 \mathrm{~h}$ at $120 \mathrm{~V}$. The membrane was incubated with mouse anti-His-Tag antibody at a dilution of 1:1,000 at $4{ }^{\circ} \mathrm{C}$ overnight, and washed three times for $15 \mathrm{~min}$ each in PBS (phosphate-buffered saline). Subsequently, the membrane was incubated with goat anti-mouse IgG antibody conjugated with HRP (horseradish peroxidase) (Boster, China) at a dilution of 1:500 for $3 \mathrm{~h}$ at $37{ }^{\circ} \mathrm{C}$. After washing twice in PBST, the membrane was displayed (TIANGEN, China) according to the manufacturer's protocol.

\subsection{Purification and Antimicrobial Activity Assays of ZmSCP in Vitro}

The pET-32a-ZmSCP constructs contained a His-tag downstream of the target gene to allow purification of the over-expressed proteins with the His-Bind kit, following the manufacturer's protocol (NovaGen, USA). The induced bacterial cell pellet was collected, lysed, and purified. The recombinant protein in $1 \times$ elution buffer was dialysed against $1 \times$ PBS (phosphate-buffered saline) with three changes at $4{ }^{\circ} \mathrm{C}$ over $24 \mathrm{~h}$. The protein concentration was measured according to Bradford et al. [18] and antimicrobial activity was evaluated in vitro.

Rhizoctonia solani was cultured on PDA p (otato dextrose agar) medium at $28{ }^{\circ} \mathrm{C}$. Holes $1 \mathrm{~cm}$ in diameter were placed on PDA plates $(9.0 \mathrm{~cm})$.
Differing concentrations of ZmSCP protein in $1 \times$ PBS buffer were added into the three holes $(20,40$, and $60 \mu \mathrm{g}$ ), with $1 \times$ PBS buffer as control. The plates were incubated at $28{ }^{\circ} \mathrm{C}$ for $48 \mathrm{~h}$. In addition, Rhizoctonia solani were incubated on PDA (potato dextrose agar) with PBS buffer (CK) or the purified protein at a concentration of $20 \mu \mathrm{g}, 40 \mu \mathrm{g}$, and $60 \mu \mathrm{g}$ $(\mathrm{ZmSCP})$ at $28{ }^{\circ} \mathrm{C}$. Germination and growth of Rhizoctonia solani were examined at $48 \mathrm{~h}$ of incubation under a microscope. The experiment was carried out three times independently, using the same conditions.

\section{Results}

\subsection{Isolation of ZmSCP and Sequence Analysis}

A specific 999 bp fragment named ZmSCP was amplified with gene specific primers. The ExPASy translation tool (http://au.expasy.org/tools/dna.html) was used to deduce the amino acid sequence of the cDNA, resulting in a sequence of 332 amino acids (Fig. 1). Further, the results indicated an expected acidic protein of $36.5 \mathrm{kD}$ with an isoelectric point of 4.75 Amino acid sequence analysis revealed that there are four types of amino acids in ZmSCP, they are hydrophobic amino acids, hydrophilic amino acids, basic amino acids, acidic amino acids and the percentages are $43.1 \%, 26.9 \%, 13.9 \%, \quad 13.1 \%$ respectively (Table 1). Phylogenetic analysis revealed

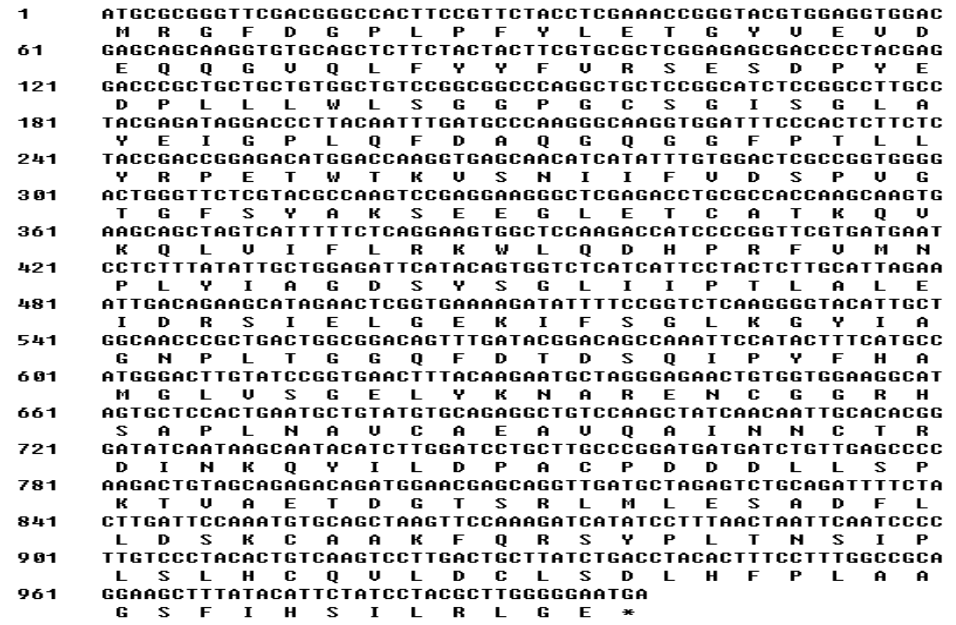

Fig. 1 Encoding amino acid sequence of $\mathrm{ZmSCP}$. 
Table 1 Amino acid compositions of $\mathrm{ZmSCP}$.

\begin{tabular}{|c|c|c|c|}
\hline Types & Percentage $(\%)$ & Name & Quantity \\
\hline \multirow{8}{*}{ Hydrophobic amino acids } & \multirow{8}{*}{43.10} & Ala & 21 \\
\hline & & Ile & 19 \\
\hline & & Leu & 41 \\
\hline & & Met & 4 \\
\hline & & Phe & 17 \\
\hline & & Pro & 21 \\
\hline & & Try & 3 \\
\hline & & Val & 15 \\
\hline \multirow{7}{*}{ Hydrophilic amino acids } & \multirow{7}{*}{29.90} & Asp & 10 \\
\hline & & Cys & 9 \\
\hline & & Glu & 15 \\
\hline & & Gly & 31 \\
\hline & & Ser & 26 \\
\hline & & Thr & 15 \\
\hline & & Tyr & 15 \\
\hline \multirow{3}{*}{ Basic amino acids } & \multirow{3}{*}{13.90} & Arg & 12 \\
\hline & & His & 6 \\
\hline & & Lys & 12 \\
\hline \multirow{2}{*}{ Acidic amino acids } & \multirow{2}{*}{13.10} & Asp & 21 \\
\hline & & Glu & 19 \\
\hline
\end{tabular}

that ZmSCP has closer kinship with Oryza satiua and sorghum, which belong to the same evolutionary branch (Fig. 2).

\subsection{Construction of Recombinant Plasmid pET32a(+)-ZmSCP}

The pMD18-ZmSCP and the pET32a(+) were double digestion with EcoR I and Not I . Competent cells $\mathrm{DH} 5 \alpha$ in E. coli were transformed by ligation products. Fourty-eight monoclonals were picked for PCR detection. Results indicate that all the 48 monoclonals were positive. The sizes of fragments after single and double enzyme digested were correct (Figs. 3a and 3b). The cDNA sequence of inserted reading frame was confirmed by sequencing. No frameshift mutation occurred indicated that the recombinant expression plasmid pET32a(+)-ZmSCP was built successfully.

\subsection{SDS-PAGE and Western Blot Analysis}

E. coli BL21 cells were transformed and expression conditions were optimized at $37{ }^{\circ} \mathrm{C}$ and $28{ }^{\circ} \mathrm{C}$, after induction with $0,0.2,0.4,0.6,0.8$, or $1.0 \mathrm{mM}$ IPTG for various time periods, $0,2,4,6,8$, and $10 \mathrm{~h}$, respectively. The mRNA encoding ZmSCP was fused to His-Tag coding sequence at the 3' end, for comparison to cells containing recombinant plasmid without induction. Results showed a distinguishable extra band around $58 \mathrm{kD}$, the size of the ZmSCP-thioredoxin fusion protein in E. coli. The optimal induction occured at $28{ }^{\circ} \mathrm{C}$ with $0.4 \mathrm{mM}$ IPTG for $4 \mathrm{~h}$ (Fig. 4).

The recombinant plasmid was transformed into the

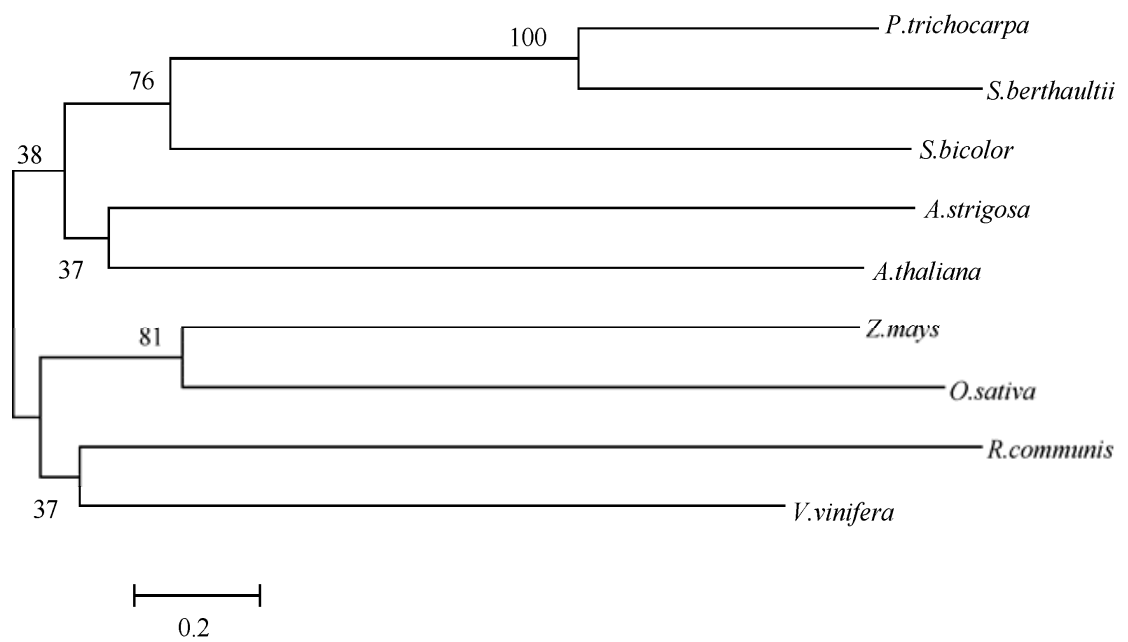

Fig. 2 Phylogenetic analysis of ZmSCP and other SCP from higher plants. Sequences were downloaded from GenBank and have the following accession numbers: P. trichocarpa (XM_002298750); S. berthaultii (AF006078); S. bicolor (XM_002446512); A. Strigosa (XM_002265806); A. Thaliana (NM_123009); O. sativa (NM_001074392); R. communis (XM_002525247); V. Vinifera (XM_002265806). 


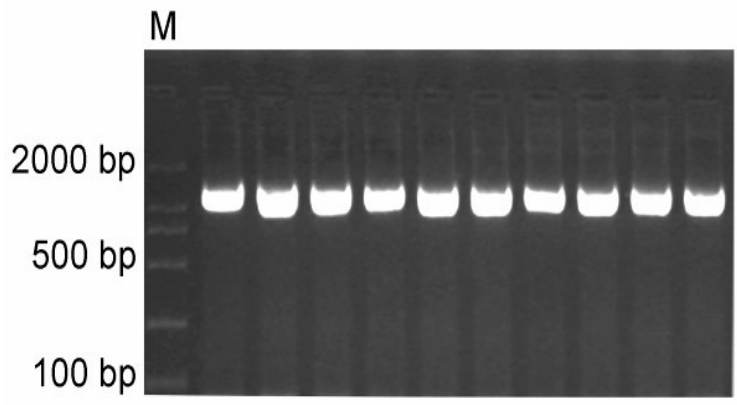

(a)

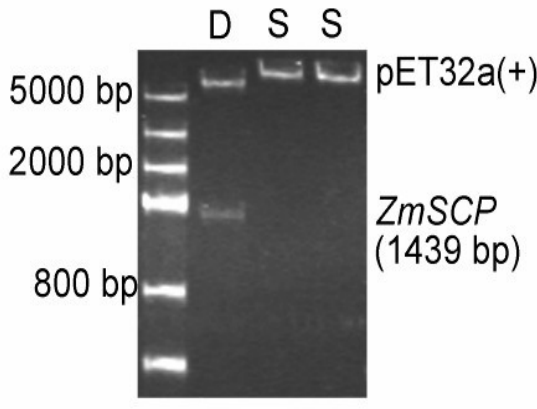

(b)

Fig. 3 Identification of recombinant plasmid pET32a(+)-ZmSCP. (a) Results of PCR with the template of plasmid; (b) Detection of double restriction enzyme digestion of plasmid pET32a(+)-ZmSCP. D stands for double restriction enzyme digestion, $S$ stands for double restriction enzyme digestion.

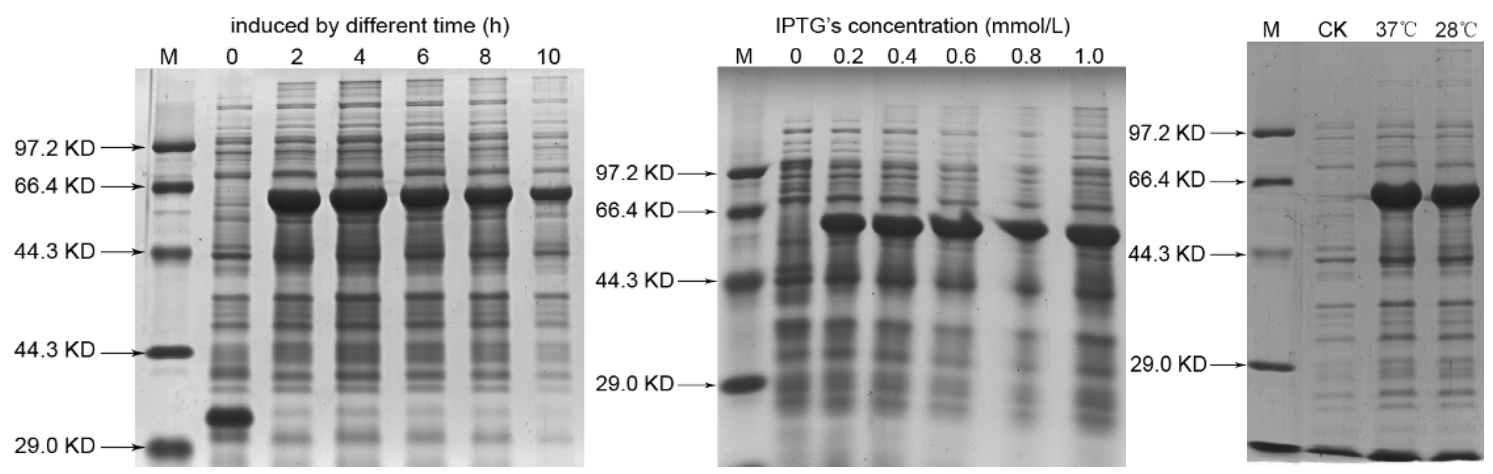

Fig.4 SDS-PAGE of optimization of prokaryotic expression system.

host strain BL21 (DE3) and induced at $28{ }^{\circ} \mathrm{C}$ with $0.4 \mathrm{mM}$ IPTG at $4 \mathrm{~h}$. The expressed proteins were analyzed by SDS-PAGE and western blot analysis Western blot analysis revealed the presence of one protein in the induced cells with apparent molecular weight of approximately $58 \mathrm{kD}$, which is bound to the anti-His-Tag antibody (Fig. 5). For analysis of antimicrobial activity of the fusion protein in vitro, the protein was purified by SDS-PAGE, which revealed highly pure protein (Fig. 6).

\subsection{Purification and Antimicrobial Activity Assays of ZmSCP in Vitro}

The recombinant protein concentration was measured with the Bradford method. The possible antifungal activity of ZmSCP against Rhizoctonia solani was investigated in vitro with three replications. Result showed that the ZmSCP protein exhibited an inhibitory effect in vitro on hyphal growth of Rhizoctonia solani. Rhizoctonia solani treated with $\mathrm{ZmSCP}$ at $60 \mu \mathrm{g} / \mathrm{mL}$ showed significantly slower hyphal growth compared with the PBS control (Fig. 7a-7c).

\section{Discussion}

SCPs have been identified in a wide array of organisms and comprise a large family of hydrolyzing enzymes, which are believed to play roles in processing and degradation of proteins/peptides. Compared to the large number of members in the SCP family, only a few of them have been studied in detail for their biochemical activity and biological roles in

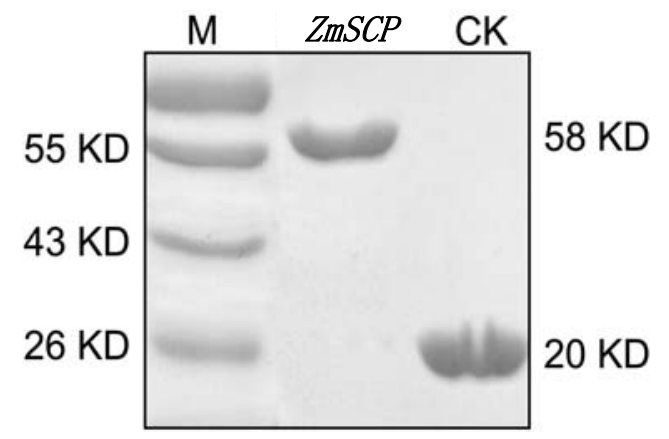

Fig.5 Identification of recombinant protein by Western Blot. 


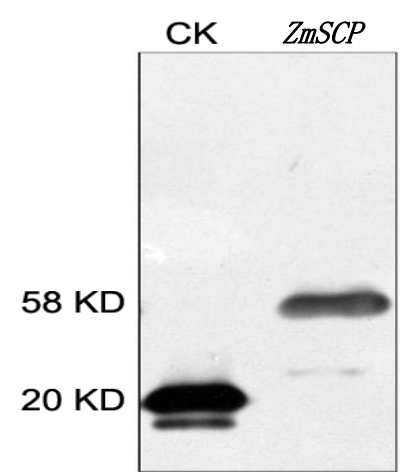

Fig. 6 SDS-PAGE analysis of purified protein.

(a)

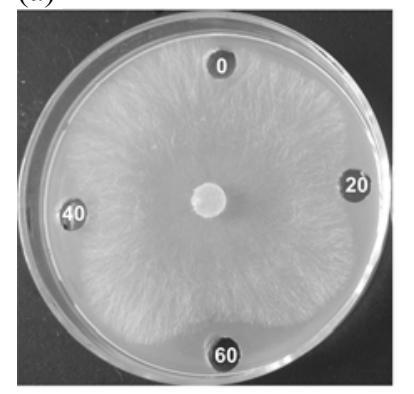

(c)

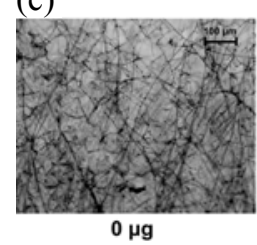

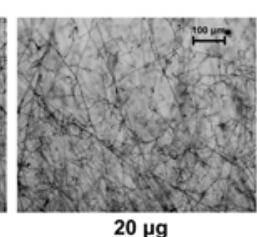

plants. OsBISCPL1 which encodes a protein containing typical conserved structural features plays roles in regulating defense responses against pathogen infection and oxidative stress. In the previous study, ZmSCP isolated from maize was up-regulated under different stress conditions [19]. In the present study, recombinant protein inhibited hyphal growth of Rhizoctonia solani, suggesting that ZmSCP plays an important role in the disease resistance response.

(b)
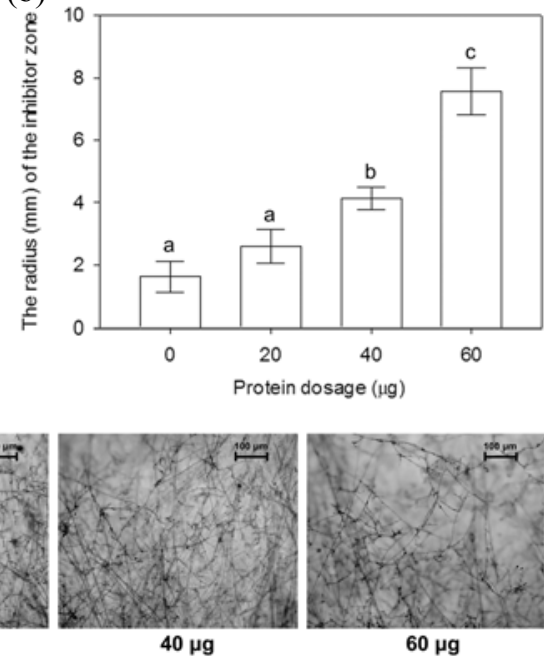

Fig. 7 Inhibitory effect of recombinant protein to Rhizoctonia solani. (a) antifungal activity of $\mathrm{ZmSCP}$ protein in culture dish; (b) statistical analysis of inhibitor zone (different letters indicate significant difference $(p<0.01)$ between treatments); (c) antifungal activity of $\mathrm{ZmSCP}$ protein under a microscope; Bars $=100 \mu \mathrm{m}$.

Many studies verified gene function using prokaryotic expressive system, for example, Lee et al. obtained a large amount of high purity expressive proteins using prokaryotic expressive system, and demonstrated the disease resistance using antimicrobial test in vitro [20]. Similar antimicrobial analyses have been conducted in studies involved rice, plantains, tobacco, and cotton [20-25]. Results of previous studies indicate part of pathogenesis-related proteins (e.g. PR2, PR3 and PR4) with antibacterial properties in different degrees [26]. The possible reason is that these pathogenesis-related proteins are extracellular proteins, and when the invasion of the pathogen occurred, pathogenesis-related proteins can quickly assembled to resistance to pathogens [26]. Although the present studies have demonstrated that
ZmSCP plays important roles in defense responses against biotic and abiotic stress, the mechanism by which ZmSCP regulates plant defense responses against biotic and abiotic stresses remains to be further explored.

\section{Conclusion}

The authors cloned and identified a new maize serine carboxypeptidase gene named ZmSCP. ZmSCP encodes a 332 amino acid protein with a predicted molecular mass of $36.5 \mathrm{kDa}$ and $\mathrm{pI}$ of 4.75 . Phylogenetic analysis revealed that ZmSCP showed closer kinship with Oryza sativa and sorghum, which belong to the same evolutionary branch. The authors subsequently purified the recombinant protein which expressed in Escherichia coli BL21 and analyzed its 
antimicrobial activities in vitro. Results showed that the recombinant protein inhibited hyphal growth of Rhizoctonia solani. The study suggests that the expression of ZmSCP is closely related to maize sheath blight resistance caused by Rhizoctonia solani.

\section{Acknowledgments}

This research was financially supported by the Natural Science Foundation (30900901), the Science and Technology Department Application Foundation of Sichuan province (2006J13-039), and the Agriculture Project of Ministry (2011ZX08003-003).

\section{References}

[1] C.M. Fraser, M.G. Thompson, A.M. Shirley, J. Ralph, J.A. Schoenherr, T. Sinlapadech, et al., Related arabidopsis serine carboxypeptidase-like sinapoylglucose acyltransferases display distinct but overlapping substrate specificities, Plant Physiology 144 (4) (2007) 986-1999.

[2] Y. Feng, Q.Z. Xue, The serine carboxypeptidase like gene family of rice (Oryza sativa L. ssp. japonica), Functional Integrative Genomic 6 (1) (2006) 14-24.

[3] J. Vendrell, F.X. Aviles. Carboxypeptidases, vol. 2, in: V. Turk (Ed.), Proteases: New Perspectives, Birkhauser-Verlag, Basel, 1999, pp. 13-34.

[4] T.L. Bullock, K. Braddam, S.J. Remington, Peptide aldehyde complexes with wheat serine carboxypeptidase II : Implications for the catalytic mechanism and substrate specificity, Journal of Molecular Biology 255 (1996) 714-725.

[5] D.S. Moura, D.R. Bergey, C.A. Ryan, Characterization and localization of a wound-inducible type 1 serine-carboxypeptidase from leaves of tomato plants, Planta 212 (2001) 222-230.

[6] M. Cercos, C. Urbez, J. Carbonc, A serine carboxypeptidase gene ( $P s C P$ ), expressed in early steps of reproductive and vegetative development in Pisum sativum, is induced by gibberellins, Plant Molecular Biology 51 (2003) 165-174.

[7] A.M. Shirley, C. Chapple, Biochemical characterization of sinapoy glucose choline sinapoyltransferase, a serine carboxypeptidase-like protein that functions as an acyltransferase in plant secondary metabolism, Biological Chemistry 278 (2003) 19870-19877.

[8] H.Z. Liu, X.E. Wang, H.J. Zhang, Y.Y. Yang, X.C. Ge, F.M. Song, A rice serine carboxypeptidase-like gene OsBISCPL1 is involved in regulation of defense responses against biotic and oxidative stress, Gene 420
(2008) 57-65.

[9] D.F. Degan, A. Rocher, V. Cameron-Mills D. von Wettstein, The expression of serine carboxypeptidases during maturation and germination of the barley grain, Proceedings of the National Academy of Sciences of the United States of American 91 (1994) 8209-8213.

[10] F. Domnguez, M.C. Gonzlez, F.J. Cejudo, A germination-related gene encoding a serine carboxypeptidase is expressed during the differentiation of the vascular tissue in wheat grains and seedlings, Planta 215 (2002) 727-734.

[11] S.J. Granat, K.A. Wison, A.L. Wilson, New serine carboxypeptidase in mung bean seedling cotyledons, Journal of Plant Physiology 160 (2003) 1263-1266.

[12] L. Jia, K.A. Lease, F.E. Tax, J.C. Walker, BRS1, a serine carboxypeptidase, regulates BRI1 signaling in Arabidopsis thaliana, Proceedings of the National Academy of Sciences of the United States of American 98 (2001) 5916-5921.

[13] C. Milkowski, D. Strack, Serine carboxypeptidase-like acyltransferases, Phytochemistry 65 (2004) 517-524.

[14] M.J. Zhao, Z.M. Zhang, S.B. Gao, W.C. Li, T.Z. Rong, G.T. Pan, Identification of quantitative trait loci controlling resistance to banded leaf-sheath blight in Maize, Acta Ggronomica Sinica 32 (2006) 698-702. (in Chinese)

[15] L. Liu, Y.Y. Ma, Z.M. Zhang, G.T. Pan, M.J. Zhao, cDNA-AFLP analysis of Rhizoctonia solani AG1-IA-induced maize differential gene expression, Acta Phytopathologica Sinica 39 (4) (2009) 385-391. (in Chinese)

[16] Z.M. Zhang, L. Liu, H.J. Lin, G.S. Yuan, X. Zeng, Y.O. Shen, et al., Identification of genes differentially expressed in maize (Zea mays L.) during Rhizoctonia Solani Kühn infection by suppression subtractive hybridization, African Journal of Biotechnology 11 (2012) 2827-2838.

[17] J. Sambrook, E.F. Fritsch, T. Maniatis, Molecular Cloning: A Laboratory Manual, Cold Spring Harbor Laboratory Press, NY, 1989.

[18] M.M. Bradford, A rapid and sensitive method for the quantitation of microgram quantities of protein utilizing the principle of protein-dye binding, Analytical Biochemistry 72 (1976) 248-254.

[19] L. Liu, Z.M. Zhang, M.J. Zhao, J. Wang, H.J. Lin, Y.O. Shen, et al., Molecular cloning and characterization of the pathogenesis related protein 5 in Zea mays and its antifungal activity against Rhizoctonia solani, African Journal of Biotechnology 10 (2011) 19286-19293.

[20] S.C. Lee, I.S. Hwang, H.W. Choi, B.K. Hwang, Involvement of the pepper antimicrobial protein CaAMP1 gene in broad spectrum disease resistance, 
Plant Physiology 148 (2008) 1004-1020.

[21] Y.L. Gao, W.Z. Guo, L. Wang, T.Z. Zhang, Isolation and characterization of resistance and defense gene analogs in cotton (Gossypium barbadense L.), Science in China Series C: Life Science 49 (6) (2006) 530-542.

[22] G.N. Wang, X.H. Ding, M. Yuan, D.Y. Qiu, Dual function of rice OsDR8 gene in disease resistance and thiamine accumulation, Plant Molecular Biology 60 (2006) 437-449.

[23] X.L. Jin, D.R. Feng, H.B. Wang, J.F. Wang, A novel tissue-specific plantain beta-1,3-glucanase gene that is regulated in response to infection by Fusarium oxysporum fsp cubense, Biotechnology Letters 29 (2007)
1431-1437.

[24] L.R. Yang, H. Sun, Z.S. Lei, X.L. Zhao, X. Quan, B.G. Xue, Cloning, prokaryotic expression and activity assay of chitinase (Tvchi) from Trichoderma viride, Acta Phytopathologica Sinica 42 (2) (2012) 139-145. (in Chinese)

[25] H.Q. Xian, W. Tang, L.Q. Zhang, A.N. Li, D.C. Li, Cloning and expression of a chitinase gene tfchil from Talaromyces flavus and the antifungal activity of the recombinant enzyme, Acta Phytopathologica Sinica 42 (2) (2012) 146-153. (in Chinese)

[26] B. Fritig, T. Heitz, M. Legrand, Antimicrobial proteins in induced plant defense, Current Opinion in Immunology 10 (1998) 16-22. 